Research Paper

\title{
Comparison of Combined Transcatheter Arterial Chemoembolization and CT-guided Radiofrequency Ablation with Surgical Resection in Patients with Hepatocellular Carcinoma within the Up-to-seven Criteria: A Multicenter Case-matched Study
}

\author{
Tao $\operatorname{Pan}^{1 *}$, Lu-Wen $\mathrm{Mu}^{2 *}$, Chun $\mathrm{Wu}^{1}$, Xi-qun $\mathrm{Wu}^{1}$, Qian-Kun $\mathrm{Xie}^{2}$, Xi-Shan $\mathrm{Li}^{3}$, Ning Lyu ${ }^{2}$, Shao-long Li ${ }^{2}$, \\ Hai-jing Deng², Zai-bo Jiang ${ }^{1}$, Ai-hua Lin ${ }^{4}$, Ming Zhao ${ }^{2 凶}$ \\ 1. Department of Vascular Interventional Radiology, the Third Affiliated Hospital, Sun Yat-sen University, Guangzhou, China; \\ 2. Division of Minimally Invasive Interventional Therapy, State Key Laboratory of Oncology in South China, Sun Yat-sen University Cancer Center, \\ Collaborative Innovation Center for Cancer Medicine, Guangzhou, China; \\ 3. Department of Interventional Radiology, Guangzhou First People's Hospital, Guangzhou Medical University, Guangzhou, China; \\ 4. School of Public Health, Sun Yat-sen University, Guangzhou, China. \\ * Tao Pan and Lu-Wen Mu are co-first authors of this study and they contributed equally to this work.
}

$\triangle$ Corresponding author: Ming Zhao, MD, PhD, Division of Minimally Invasive Interventional Therapy, Medical Imaging Center, State Key Laboratory of Oncology in South China, Sun Yat-sen University Cancer Center, Collaborative Innovation Center for Cancer Medicine, 651 Dongfeng Road East, Guangzhou 510060, P.R. China. E-mail: zhaoming@sysucc.org.cn. TEL:+86-020-8734-3272, FAX:+86-020-8734-3272

(c) Ivyspring International Publisher. This is an open access article distributed under the terms of the Creative Commons Attribution (CC BY-NC) license (https://creativecommons.org/licenses/by-nc/4.0/). See http://ivyspring.com/terms for full terms and conditions.

Received: 2017.03.07; Accepted: 2017.09.11; Published: 2017.09.29

\begin{abstract}
Background \& Aims: We compared the efficacy of transcatheter arterial chemoembolization (TACE) in combination with CT-guided radiofrequency ablation (RFA) with that of surgical resection (SR) in patients with hepatocellular carcinoma (HCC) within the up-to-seven criteria.

Methods: From January 2004 to December 2014, 420 multicenter consecutive patients with HCC who conformed to the up-to-seven criteria and initially received either TACE plus CT-guided RFA (TACE-RFA) or SR were enrolled. A matched cohort composed of 206 patients was selected after adjustment with propensity score matching. The overall survival (OS) of each patient was calculated with the Kaplan-Meier method and compared by the log-rank test.

Results: The median OS and 1-, 3-, and 5-year survival rates were 56.0 months, $96.1 \%, 76.7 \%$ and $41.3 \%$ in the TACE-RFA group and 58.0 months, $96.1 \%, 86.4 \%$ and $46.2 \%$ in the SR group, respectively. There was no significant difference in OS between the two groups $(P=0.138)$. For patients with HCC beyond the Milan criteria, TACE-RFA provided a longer median OS than SR (52.0 vs 45.0 months, $P=0.023)$.

Conclusions: Treatment by TACE-RFA conferred an OS rate comparable with that of SR in patients within the up-to-seven criteria. For patients with HCC between the Milan and the up-to-seven criteria, TACE-RFA might be superior to SR for survival prolongation.
\end{abstract}

Key words: Transarterial chemoembolization; Radiofrequency ablation; Surgical resection; Hepatocellular carcinoma; Up-to-seven criteria.

\section{Introduction}

Hepatocellular carcinoma (HCC) is the sixth most common cancer and the second most common cause of cancer death in the world [1]. Most HCCs are diagnosed at intermediate or advanced stages, and around $30 \%$ of patients with early HCC benefit from curative therapies such as liver transplantation (LT) and surgical resection (SR) [2]. However, a shortage of donors and poor hepatic reserve with liver cirrhosis 
limit the possibilities for LT and SR [3].

Radiofrequency ablation (RFA) is considered another alternative curative therapy to SR in patients with early HCC, especially in patients with impaired liver function $[3,4]$, but the relatively high local tumor progression rate resulting from incomplete ablation or progression of daughter lesions is a negative prognostic factor for patients' survival [5]. The use of transcatheter arterial chemoembolization (TACE) may reduce the local tumor progression rate arising from large ablative tumors due to the decreased blood flow and minimized heat loss; in addition, TACE is effective in diminishing the tumor dissemination [6]. The combination of TACE and RFA (TACE-RFA) has been proved to be comparable with $S R$ in OS prolongation for patients within the Milan criteria (single tumor $\leq 5 \mathrm{~cm}$ in size or $\leq 3$ tumors each $\leq 3 \mathrm{~cm}$ in size, and no macrovascular invasion) [7, 8], and also led to good results for patients with HCCs exceeding the Milan criteria in many cancer centers $[6,9,10]$. Some oncologists have suggested that patients within the up-to-seven criteria (HCCs with 7 as the sum of the size of the largest tumor [in $\mathrm{cm}$ ] and the number of tumors) might be eligible for TACE-RFA treatment [11], while some other researchers have recommended SR for these patients because most of them have resectable disease and the postoperative survival time was promising [12]. The difference between the survival benefits of these two therapeutic modalities remains unclear. Moreover, with the merit of high resolution and without interference of gas

Consecutive HCC patients in 2 centers during 10 years $(n=15260)$

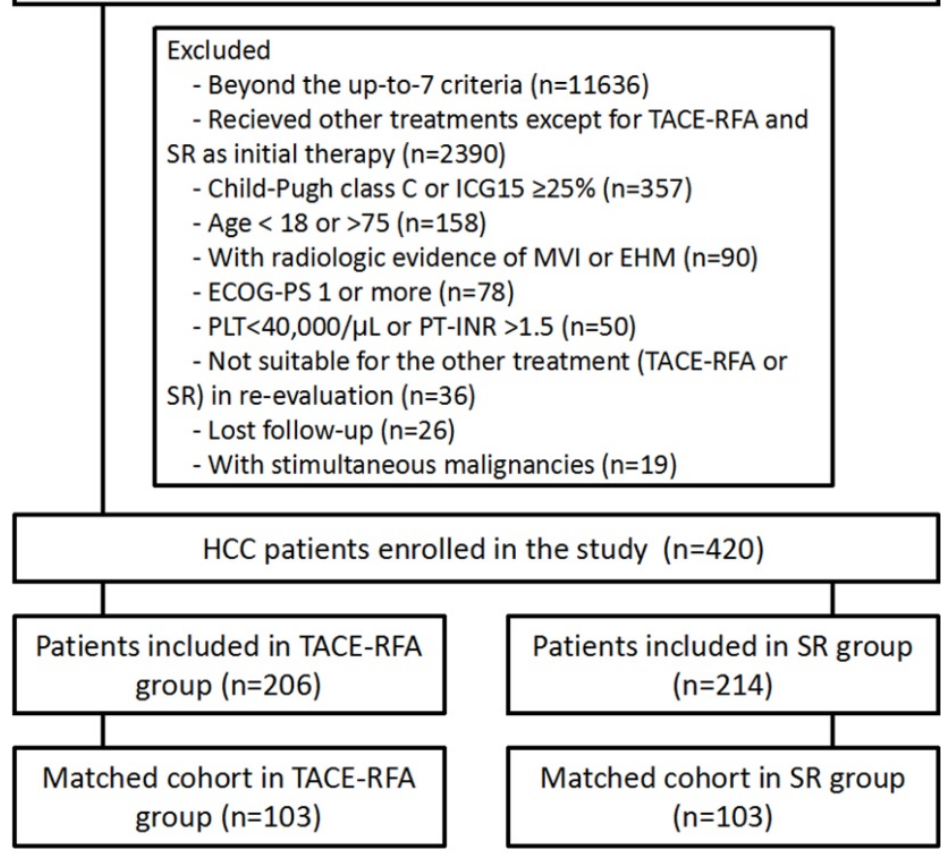

Figure 1. Flow diagram showing patient selection criteria. released during the procedure, the use of $\mathrm{CT}$ guidance in the RFA procedure helps operators to ablate even large tumors with a safety margin greater than that with the widely used ultrasonographic guidance [6, 13]. The aim of this work was to compare the safety and efficacy of TACE in combination with available CT-guided RFA with that of SR in patients with HCC who met the up-to-seven criteria in a large multicenter case-matched study.

\section{Patients and Methods}

\section{Patient selection}

The study was approved by the ethical committee of our institutions (Sun Yat-sen university cancer center and the third affiliated hospital of Sun Yat-sen university). From our prospectively maintained electronic database from January 2004 to December 2014, we retrospectively enrolled 420 patients according to the predefined eligibility and exclusion criteria. The eligibility criteria were as follows: 1) age 18 to 75 years; 2) an HCC diagnosis confirmed by pathology or diagnosed by dynamic CT or MRI showing intense arterial uptake followed by washout of contrast in the venous-delayed phases to patients with cirrhosis or chronic hepatitis B [2]; 3) treatment by SR or the combination of TACE and CT-guided RFA as the initial treatment, and suitability for the other treatment modality in a re-evaluation by the researchers; 4) HCC classified within the up-to-seven criteria at the time of the initial treatment; 5) no radiologic evidence of invasion into major portal/hepatic venous branches and no extrahepatic metastases; 6) cirrhosis classified as Child-Pugh class A or B with $<25 \%$ retention of indocyanine green 15 min after injection. The exclusion criteria were as follows: 1) an Eastern Cooperative Oncology Group performance status of 1 or more; 2) presence of simultaneous malignancies; 3) lost to follow-up; 4) platelet count $<40,000 / \mu \mathrm{L}$; and 5) an international normalized ratio $>1.5$.

The patients enrolled were divided into two groups: 214 patients who underwent resection were assigned to the SR group, and another 206 patients treated by TACE plus RFA were included in the TACE-RFA group. The flow chart of patient selection is shown in Figure 1.

\section{Treatment procedures}

The treatment modality of each patient was decided by a HCC multidisciplinary treatment team that included hepatobiliary surgeons, medical 
oncologists, interventional radiologists, and diagnostic radiologists. For patients with HCC within the up-to-seven criteria, TACE-RFA and SR were the two most common treatment modalities routinely applied at our centers.

The TACE and RFA procedures have been described in previous works $[6,14,15]$. They were performed by three intervention oncologists (M.Z, Z.B.J, C.W), who had more than 10 years of experience with interventional techniques. The TACE procedure was performed in all the patients in the TACE-RFA group by using super-selective catheterization to inject an emulsion of 8-15 $\mathrm{ml}$ of lipiodol (Laboratorie Guerbet, ulnay-sous-Bois, France), 40-60 mg of epirubicin (Farmorubicin; Pharmacia, Tokyo, Japan), and 6-10 mg of mitomycin C (Kyowa Hakko Kogyo, Tokyo, Japan), followed by embolization with gelatin sponge particles (Gelfoam; Hangzhou, China) into the tumors. Within 2 weeks (median, 6 days; range, 2-14 days) after TACE, the RFA procedure was performed percutaneously with CT-guidance (Siemens, Munich, Germany). We used a single internally cooled electrode with a 2- or 3-cm active tip chosen according to the tumor size (Cool-Tip, Valleylab, MA, USA or Star RF electrode, Starmed, Gyeonggi-do, Korea) after administration of conscious sedatives and local anesthesia. RFA was then applied with an impedance control algorithm for 10-20 min per RFA application and was targeted to tumors shown in contrast-enhanced CT to achieve an overlapping safety margin of 5-10 $\mathrm{mm}$.

Under general anesthesia, SR was performed by experienced surgeons (4 nonauthors), who had between 13 and 21 years of experience with hepatectomy. An intraoperative ultrasound was routinely used to evaluate the tumor extent, the liver remnant, and the possibility of a negative resection margin. An anatomical resection of the HCC, aiming at a resection margin of at least $10 \mathrm{~mm}$, was performed in the form of segmentectomy and/or subsegmentectomy as described by Makuuchi et al. [16], that considered tumor extent and hepatic functional reserve.

\section{Follow-up}

The follow-up period was defined as the duration from the date of initial treatment until death or the last visit by July 30, 2016. According to the routine follow-up protocol of our centers, 4-phase contrast-enhanced abdominal CT or MRI images were obtained every 2 to 3 months for the first year and every 6 months thereafter. In the TACE-RFA group, up to 2 additional RFA treatments were performed for patients with residual tumor after the initial RFA; the interval between 2 RFA treatments was 1 month. If intrahepatic tumor recurrence or distant metastases were present in both groups at follow-up, subsequent treatments including resection, RFA, TACE, sorafenib, systemic chemotherapy, and best supportive care were applied after discussion by the multidisciplinary teams of each center. Antiviral treatment was routinely performed in patients with hepatitis virus infection.

The main outcome of this study was OS, which was defined as the time interval from the first treatment to the date of death from any cause or to the last visit. Other outcomes included the following: 1) local tumor progression rate, defined as the rate of appearance over follow-up of foci of untreated disease in tumors that were previously considered to be completely ablated or resected; 2) independent prognostic factors associated with OS; 3) treatment-related death and major complication rates (a major complication was defined as an event that led to substantial morbidity and disability, an increase in the level of care, or resulted in a hospital admission or which substantially lengthened the hospital stay [17]).

\section{Statistical analysis}

Statistical analyses were performed by an experienced medical statistician (A.H.L) using SPSS 20.0 software (SPSS, Chicago, IL). The two groups were compared using Student's $t$ test for continuous data and the chi-square test for categorical data. To minimize the potential selection bias, propensity score-matching analyses were generated using binary logistic regression. Independent variables were entered into the propensity model, including sex, age, tumor number, and size, Milan criteria, platelet counts, Child-Pugh classification, hepatitis B surface antigen status, levels of serum a-fetoprotein (AFP), alanine aminotransferase (ALT), albumin (ALB), and total bilirubin (TB). One-to-one matching between the groups was accomplished using the nearest-neighbor matching method (caliper $=0.1$ ). Matched data were analyzed using the paired $t$ test or the Wilcoxon signed-rank test for continuous variables and the McNemar test for categorical variables. Cumulative OS was calculated using the Kaplan-Meier method and was compared using the log-rank test. The independent prognostic factors related to OS were assessed using multivariate Cox proportional hazards regression analysis. All baseline variables listed in Table 1 were included in the univariate analysis, and the variables with statistical significance were analyzed in the multivariate analysis to assess their significance as independent predictors. Statistical tests were two-sided, and $P<.05$ was considered to indicate a significant difference. 
Table 1. Baseline Characteristics of Patients Who Underwent Surgical Resection or TACE-RFA before and after Propensity Score Matching.

\begin{tabular}{|c|c|c|c|c|c|c|}
\hline \multirow[t]{2}{*}{ Characteristic } & \multicolumn{3}{|l|}{ All patients $(n=420)$} & \multicolumn{3}{|c|}{ Matched cohort $(n=206)$} \\
\hline & $\begin{array}{l}\text { SR Group } \\
(n=214, \%)\end{array}$ & $\begin{array}{l}\text { TACE-RFA Group } \\
(n=206, \%)\end{array}$ & $P$ Value & $\begin{array}{l}\text { SR Group } \\
(n=103, \%)\end{array}$ & $\begin{array}{l}\text { TACE-RFA Group } \\
(n=103, \%)\end{array}$ & $P$ Value \\
\hline Age & $53.8 \pm 11.6$ & $55.6 \pm 11.3$ & .100 & $54.6 \pm 11.0$ & $54.8 \pm 10.9$ & .477 \\
\hline $\operatorname{Sex}(M / F)$ & $203 / 11(94.9 / 5.1)$ & $190 / 16(92.2 / 7.8)$ & .273 & $97 / 6(94.2 / 5.8)$ & $95 / 8(92.2 / 7.8)$ & .593 \\
\hline HBsAg (positive/negative) & $184 / 30(86.0 / 14.0)$ & $167 / 39(81.1 / 18.9)$ & .179 & $91 / 12(88.3 / 11.7)$ & $87 / 16(84.5 / 15.5)$ & .450 \\
\hline Tumor number $(1 />1)$ & $176 / 38(82.2 / 17.8)$ & $166 / 40(80.6 / 19.4)$ & .662 & $75 / 28(72.8 / 27.2)$ & $71 / 32(68.9 / 31.1)$ & .606 \\
\hline Tumor size $(\leq 3 / 3-5 />5 \mathrm{~cm})$ & $\begin{array}{l}41 / 133 / 40 \\
(19.2 / 62.1 / 18.7)\end{array}$ & $\begin{array}{l}42 / 119 / 45 \\
(20.4 / 57.8 / 21.8)\end{array}$ & .421 & $\begin{array}{l}24 / 52 / 27 \\
(23.3 / 50.5 / 26.2)\end{array}$ & $\begin{array}{l}21 / 60 / 22 \\
(20.4 / 58.2 / 21.4)\end{array}$ & .475 \\
\hline Milan criteria (within/beyond) & $130 / 74(60.7 / 39.3)$ & $151 / 65(73.3 / 26.7)$ & .178 & $69 / 34(67.0 / 33.0)$ & $65 / 38(63.1 / 36.9)$ & 637 \\
\hline Child-Pugh class (A/B) & $212 / 2(99.1 / 0.9)$ & $190 / 16(92.2 / 7.8)$ & .011 & $101 / 2(98.0 / 2.0)$ & $100 / 3(97.1 / 2.9)$ & .655 \\
\hline Ascites (absent/present) & $212 / 2(99.1 / 0.9)$ & $201 / 5(97.6 / 2.4)$ & .760 & $101 / 2(98.0 / 2.0)$ & $101 / 2(98.0 / 2.0)$ & $>.99$ \\
\hline AFP level $(>20 / \leq 20 \mathrm{ng} / \mathrm{mL})$ & $119 / 95(55.6 / 44.4)$ & $81 / 125(39.3 / 60.7)$ & .001 & $42 / 61(40.8 / 59.2)$ & $56 / 47(54.4 / 45.6)$ & .178 \\
\hline Albumin $(\mathrm{g} / \mathrm{L})$ & $41.7 \pm 4.4$ & $40.9 \pm 6.2$ & .156 & $41.7 \pm 3.8$ & $41.0 \pm 5.9$ & .419 \\
\hline Total Bilirubin $(\mu \mathrm{mol} / \mathrm{L})$ & $14.9 \pm 6.1$ & $15.9 \pm 8.5$ & .261 & $14.3 \pm 4.8$ & $14.9 \pm 9.3$ & .264 \\
\hline ALT level $(\mathrm{U} / \mathrm{L})$ & $59.7 \pm 54.6$ & $68.6 \pm 74.8$ & .231 & $59.9 \pm 48.3$ & $60.5 \pm 49.0$ & .390 \\
\hline Platelets counts $\left(10^{9} / \mathrm{L}\right)$ & $149.8 \pm 54.8$ & $125.6 \pm 62.5$ & $<.001$ & $133.6 \pm 50.8$ & $132.2 \pm 51.4$ & .218 \\
\hline Institution (SYSUCC/TAHSYSU) & $159 / 55(74.3 / 25.7)$ & $153 / 53(74.3 / 25.7)$ & .995 & $73 / 30(70.9 / 29.1)$ & $70 / 33(68.0 / 32.0)$ & .705 \\
\hline
\end{tabular}

SR, surgical resection; TACE, transarterial chemoembolization; RFA, radiofrequency ablation; ALT, alanine aminotransferase; AFP, alpha-fetoprotein; SYSUCC, Sun Yat-sen university cancer center; TAHSYSU, the third affiliated hospital of Sun Yat-sen university.

\section{Results}

\section{Study population}

All the 420 patients enrolled were diagnosed with HCC within the up-to-seven criteria, and HCC was diagnosed from pathological tests in $362(86.2 \%)$ patients and clinically in $58(13.8 \%)$ patients. Patients' baseline characteristics are summarized in Table 1. There were no significant differences between the TACE-RFA group and the SR group with regard to age, gender, etiology, tumor number and size, Milan criteria, institution, and levels of serum ALT, ALB and TB. The TACE-RFA group had lower PLT counts $(P<$ $0.001)$ and poorer Child-Pugh class $(P=0.011)$ than the SR group, while the SR group had a significantly higher AFP level $(P=0.001)$ than the TACE-RFA group. In the 103 pairs of patients selected by the propensity score-matching, there was no significant difference in any of the variables listed in Table 1 between the two groups.

\section{Overall survival}

The median follow-up period was 60.0 months (range, 8.4-150.0 months) in the SR group and 63.8 months in the TACE-RFA group (range, 11.3-150.0 months). During the study period, 190 patients $(88.8 \%)$ in the SR group and 184 patients $(89.3 \%)$ in the TACE-RFA group died. The median OS and the 1-, 3-, and 5-year survival rates were 61.0 months, $94.4 \%$, $83.6 \%$ and $49.5 \%$ in the SR group and 55.0 months, $95.6 \%, 80.8 \%$ and $40.6 \%$ in the TACE-RFA group, respectively. The OS in the SR group was significantly prolonged compared to the TACE-RFA group for all patients $(P=0.004)$ (Figure 2a); however, in the propensity score-matched cohort, the SR group showed no significant difference in the OS compared to the TACE-RFA group $(P=.261)$. The median OS and the 1-, 3-, and 5-year survival rates were 58.0 months, $96.1 \%, 86.4 \%$ and $46.2 \%$ in the SR group and 56.0 months, $96.1 \%, 76.7 \%$ and $41.3 \%$ in the TACE-RFA group, respectively (Figure $2 b$ ).

Subgroup analyses were performed in different stratification groups, including age, gender, etiology, tumor number and size, Milan criteria, institution, levels of serum albumin, alanine aminotransferase, total bilirubin, and platelets counts. There was no significant difference in the OS between the TACE-RFA group and the SR group in most of the stratification groups above except for the patients with HCC beyond the Milan criteria; the OS of patients with HCC beyond the Milan criteria in the TACE-RFA group was significantly prolonged compared to the SR group (median OS: 52.0 months vs 45.0 months, $P=.023$ ) (Figure 3 ).

Factors found to be associated with OS in the univariate analysis were Milan criteria (Hazard Ratio $(\mathrm{HR})=1.809, P<0.001)$ and tumor size $(\leq 5 />5 \mathrm{~cm})$ $(\mathrm{HR}=1.375, P=0.045)$. For the multivariate analysis, only the Milan criteria $(\mathrm{HR}=2.093, P<0.001)$ was analyzed as the independent prognostic factor related to OS (Table 2).

\section{Technical success and tumor progression}

Technical success was achieved in all patients of both the TACE-RFA group and SR group in the matched cohort. A total of 118 RFA sessions were performed in 103 patients. One RFA session was performed in $90(87.4 \%)$ patients, two RFA sessions were performed in $11(10.7 \%)$ patients, and three RFA sessions were performed in $2(1.9 \%)$ patients. In the SR group, anatomical resection was performed in 46 
patients $(44.7 \%)$ including trisegmentectomy, lobectomy, segmentectomy, and subsegmentectomy in 3, 8, 12, and 23 patients, respectively. Nonanatomical resection was performed for the remaining 57 patients (55.3\%).

During a median follow-up period of 53.7 months (range, 8.4-150.0 months) in the matched cohort, 79 patients $(76.7 \%)$ in the SR group and 73 patients $(71.9 \%)$ in the TACE-RFA group had tumor progression $(P=.496)$. The $1-, 3-$, and 5-year tumor progression-free survival rate was $73.8 \%, 37.2 \%$ and $24.4 \%$ in the SR group and $77.5 \%, 34.8 \%$ and $20.8 \%$ in the TACE-RFA group, respectively. Intrahepatic progression was the most common type of tumor progression, which occurred in 79 and 69 patients from the SR and TACE-RFA groups, respectively $(P=$ $0.414)$. Among patients with intrahepatic progression, 4 patients after SR and 6 patients after TACE-RFA had local tumor progressions $(P=0.754)$, while 75 patients in the SR group and 63 patients in the TACE-RFA group had intrahepatic distant progression. Another 2 patients from the SR group and 4 patients from the TACE-RFA group had tumor progression in extrahepatic organs including lungs (SR, 2; TACE-RFA, 3) and lymph nodes (TACE-RFA, 1$)(P=$ $0.436)$.

\section{Complications}

No treatment-related deaths were observed in the two groups. The major complications were liver failure in 1 patient $(1.0 \%)$ from the SR group and severe myelosuppression in 2 patients $(1.9 \%)$ from the TACE-RFA group. There was no significant difference in the major complication rate between $S R$ or TACE-RFA $(P=0.561)$.

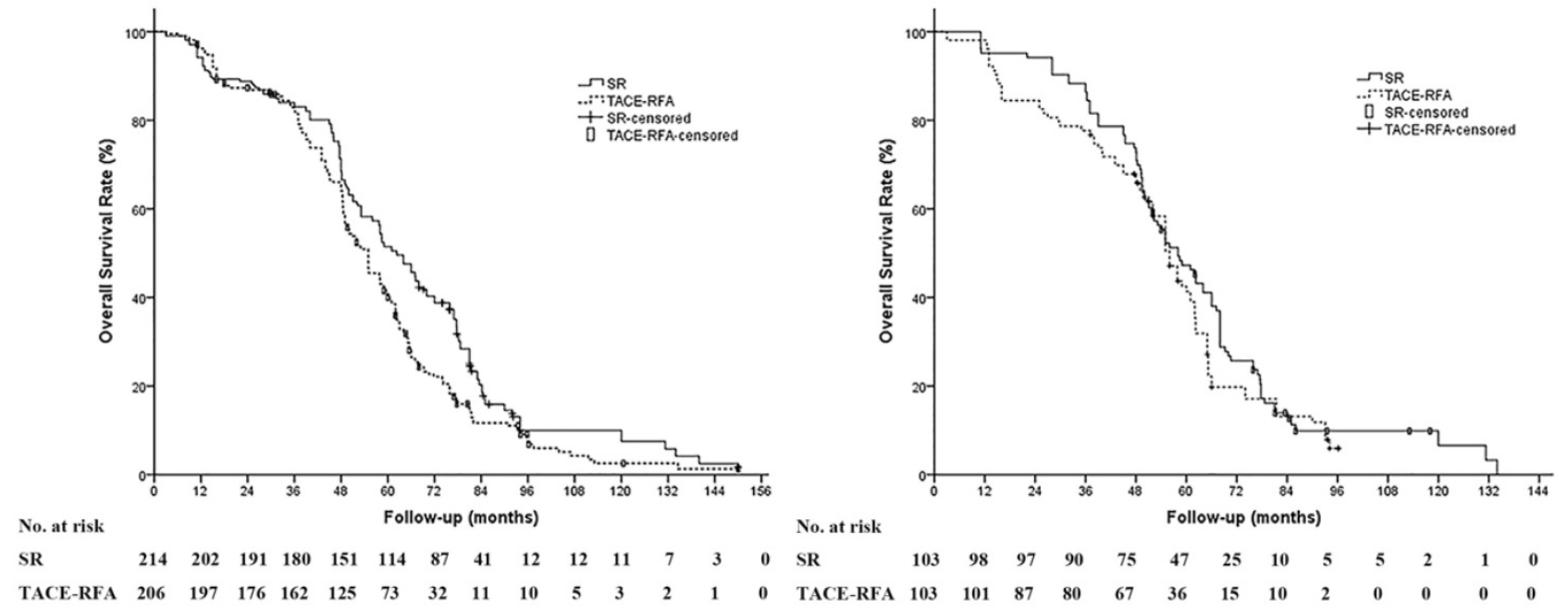

Figure 2. Overall survival (OS) of patients based on treatment modality. (A) The OS of patients in the surgical resection (SR) group was significantly prolonged compared to that in the TACE-RFA group in the whole study population (median OS: 61.0 months vs 55.0 months, $P=0.004$ ). (B) In the propensity score-matched cohort, there is no significant difference in the OS between the SR group and the TACE-RFA group (median OS: 58.0 months vs 56.0 months, $P=0.261$ ).
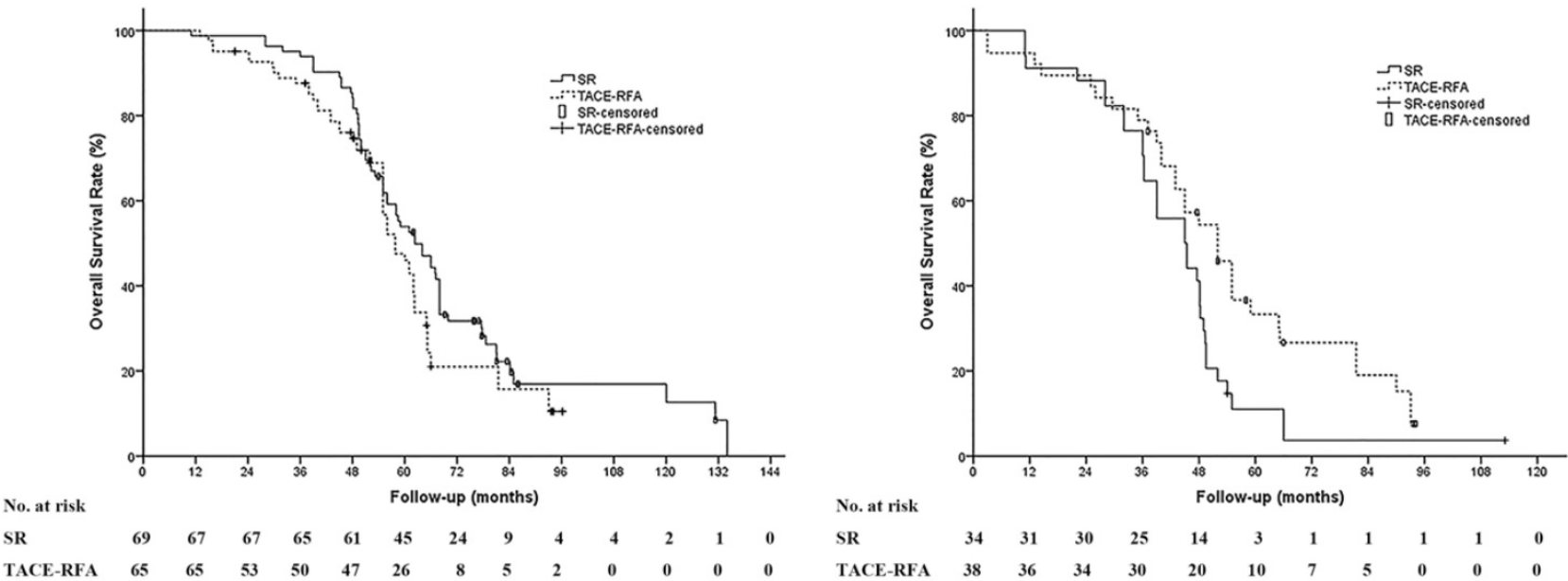

Figure 3. Subgroup analysis between patients with $\mathrm{HCC}$ within and beyond Milan criteria. (A) There was no significant difference of the OS in patients with $\mathrm{HCC}$ patients within the Milan criteria between the SR group and the TACE-RFA group (median OS: 68.3 months vs 62.0 months, $P=0.103$ ). (B) In patients with HCC beyond the Milan criteria, the OS of patients in the TACE-RFA group was significantly prolonged compared to that of the patients in the surgical resection (SR) group (median OS: 45.0 months vs 52.0 months, $P=0.023$ ). 
Table 2. Prognostic Factors Associated with OS in the Propensity Score-matched Cohort

\begin{tabular}{|c|c|c|c|c|c|}
\hline \multirow[t]{2}{*}{ Variable } & \multirow[t]{2}{*}{ No. of cases } & \multicolumn{2}{|l|}{ Univariate analysis } & \multicolumn{2}{|l|}{ Multivariate analysis } \\
\hline & & HR (95\% confidence interval) & $P$ value & HR (95\% confidence interval) & $P$ value \\
\hline Age (y) $(<70 / \geq 70)$ & $164 / 42$ & $1.485(0.932-2.354)$ & .091 & & \\
\hline $\operatorname{Sex}(M / F)$ & $192 / 14$ & $0.959(0.559-1.646)$ & .880 & & \\
\hline HBsAg (positive/negative) & $178 / 28$ & $1.358(0.957-1.928)$ & .087 & & \\
\hline Tumor number $(1 />1)$ & $146 / 60$ & $1.282(0.981-1.676)$ & .069 & & \\
\hline Tumor size $(\leq 5 />5 \mathrm{~cm})$ & $157 / 49$ & $1.375(1.007-1.878)$ & .045 & $1.321(0.894-1.953)$ & .163 \\
\hline Milan criteria (In/Out) & $134 / 72$ & $1.809(1.407-2.325)$ & $<.001$ & $2.093(1.526-2.869)$ & $<.001$ \\
\hline Child-Pugh A/B & $201 / 5$ & $1.135(0.601-2.150)$ & .881 & & \\
\hline $\operatorname{AFP}(n g / m L)(<20 / \geq 20)$ & $98 / 108$ & $1.002(0.769-1.304)$ & .990 & & \\
\hline Treatment group (SR/TACE-RFA) & $103 / 103$ & $1.182(0.820-1.683)$ & .379 & & \\
\hline $\operatorname{PLT}\left(10^{9} / \mathrm{L}\right)(<100 / \geq 100)$ & $67 / 139$ & $0.779(0.586-1.037)$ & .087 & & \\
\hline Institution (SYSUCC/TAHSYSU) & $143 / 63$ & 1.347 (0.821-2.174) & .228 & & \\
\hline
\end{tabular}

HR, hazard ratio; AFP, alpha-fetoprotein; PLT, Platelets; SR, surgical resection; TACE, transarterial chemoembolization; RFA, radiofrequency ablation; SYSUCC, Sun Yat-sen university cancer center; TAHSYSU, the third affiliated hospital of Sun Yat-sen university.

\section{Discussion}

Previous studies have reported the local tumor progression rate after RFA alone in early stage HCC as high as $15.2-41.0 \%$ over median follow-up periods ranging from 16.0 to 38.0 months [18-20]. In comparison, the local tumor progression rates after TACE-RFA have generally been lower than those of RFA alone, with previously published rates of $2.9 \%-14.5 \%$ over median follow-up periods ranging from 37.0-50.0 months [7, 20, 21]. In our study, for patients within the up-to-seven criteria, the local tumor progression rate of $5.8 \% \quad(6 / 103)$ after TACE-RFA was comparable with previous reports, and there was no significant difference from the rate after SR. Treatment with TACE in combination with CT-guided RFA may improve local tumor control via several mechanisms. First, performing TACE before RFA could reduce the viable tumor volume and decrease the "heat-sink" effect, and thus facilitate to obtain a safety ablation margin for large tumors [8, 21]. Second, digital subtraction angiography during the TACE procedure might be helpful in detecting possible micro-satellite lesions and intrahepatic micro-metastases, and moreover, TACE has been shown to be effective in controlling these micro-tumors because of the long-term anti-cancer drug release in the liver $[6,22]$. Third, the use of CT-guidance during ablation in particular could show post-TACE lipiodol deposition clearly, and thus improve accurate tumor positioning and allow an ablation safety margin while maintaining the greatest liver parenchymal salvage. Under high-resolution CT-guidance, operators could accurately use overlapping techniques for large tumors, which might achieve similar effects to the no-touch multibipolar RFA [23]. Thus, our results confirmed that combined TACE and CT-guided RFA could reduce the local tumor progression rate in patients with small or medium-sized HCCs.

In this study, the probabilities of OS at 1, 3, and 5 years in patients with HCC within the up-to-seven criteria were similar between the TACE-RFA and SR groups $(96.1 \%, 76.7 \%$ and $41.3 \%$ versus $96.1 \%, 86.4 \%$ and $46.2 \%$, respectively). Our result confirmed the conclusion of some previous studies that TACE-RFA confers a comparable OS with SR [8, 21]; in addition, as these studies enrolled patients with HCC within the Milan criteria, our study compared these 2 treatments in a larger population for the first time. The 5-year OS rate of our patients was a little lower than that of patients with early stage HCC who underwent TACE-RFA or SR, which were as high as $58 \%-75 \%$ and $56 \%-81 \%$, respectively $[8,21]$. The main reason might be the difference in patients' tumor stage; about $35 \%$ of our enrolled patients have HCC beyond the Milan criteria, and they have relatively lower OS than those within the Milan criteria. This was in accordance with our result from multivariate analysis, which indicated that HCC exceeding the Milan criteria was the only poor prognostic factor related to OS.

In our study, a set of patients with HCC beyond the Milan criteria but not exceeding the up-to-seven criteria had relatively lower tumor burden and were classified as BCLC B1 stage of HCC as described by Bolondi and his colleagues [11]. As the standard care, TACE alone conferred a modest complete tumor necrosis rate and survival benefit, while these patients were latent for curative therapy. Some studies have shown that aggressive SR could provide longer OS than TACE alone for resectable HCC patients beyond the Milan criteria (BCLC B stage) [24, 25]. Similarly, TACE-RFA has been reported to have a superior survival benefit to TACE or RFA alone in some patients with BCLC B stage HCC [22]. We observed that TACE-RFA confers longer OS than that after SR in our patients. The potential reason might be as follows: First, for single large or multifocal HCCs, the risk of intrahepatic metastasis was significantly higher than that of small HCCs, and treatment with TACE seems superior to SR in marking and 
inactivating the undetected intrahepatic metastasis [26]. Second, TACE-RFA treatment could diminish liver damage caused by repeated TACE, because only one TACE per patient was needed. Third, for multifocal HCCs or HCCs located deep in the liver, SR seemed to be inferior to TACE-RFA because more normal liver needed to be removed, and therefore there was more liver storage loss [27]. Our finding supported the proposal of an intermediate HCC subclassification, which recommended TACE-RFA for the treatment of patients with HCC patients within the up-to-seven criteria [11].

In our study, there were no treatment-related deaths, and the major complication rates in both treatment groups were low $(1.0 \%$ and $1.9 \%$ in the SR and TACE-RFA groups, respectively). Our results were similar to previous reports of early HCC treated with TACE-RFA and SR, which have major complications rates of $0 \%-2.2 \%$ and $0 \%-3.2 \%$, respectively $[7,21]$. All these studies confirmed the safety of both SR and TACE-RFA.

There are a few potential limitations of this study. Because of its retrospective nature and relatively small sample size, our study is subject to selection biases. In addition, it is clear that for tumors larger than $3 \mathrm{~cm}$, there is a benefit from combination therapy, but it is not clear whether adding TACE to RFA is really beneficial for tumors smaller than $3 \mathrm{~cm}$ [28].

\section{Conclusion}

Our study indicated that TACE combined with CT-guided RFA is safe and may confer an OS rate comparable with that of SR for patients with HCC within the up-to-seven criteria. TACE and CT-guided RFA might provide a longer OS than SR for HCC patients between the Milan criteria and the up-to-seven criteria. Although these findings should be confirmed in prospective randomized controlled trials, our analyses suggest that TACE-RFA may be considered an alternative treatment modality when SR is not feasible.

\section{Acknowledgments}

This work was not supported by any finance.

\section{Author Contributions}

Study concept and design: Tao Pan, Ming Zhao;

Acquisition of data: Lu-wen Mu, Qian-kun Xie, Xi-qun $\mathrm{Wu}$;

Analysis and interpretation of data: Ai-hua Lin, Tao Pan;

Drafting of the manuscript: Tao Pan, Lu-wen $\mathrm{Mu}$;

Critical revision of the manuscript for important intellectual content: Chun Wu, Qian-kun Xie, Xi-shan Li, Ning Lyu, Shao-long Li, Hai-jing Deng;

Statistical analysis: Ai-hua Lin;

Obtained funding: Ming Zhao;

Administrative, technical, or material support: Ming Zhao, Zai-bo Jiang;

Study supervision: Ming Zhao, Zai-bo Jiang.

\section{Competing Interests}

The authors have declared that no competing interest exists.

\section{References}

1. Torre LA, Bray F, Siegel RL, et al. Global cancer statistics, 2012. CA Cancer J Clin. 2015; 65: 87-108.

2. Bruix J, Sherman M. Management of hepatocellular carcinoma: an update. Hepatology. 2011; 53: 1020-1022.

3. Bruix J, Reig M, Sherman M. Evidence-based diagnosis, staging, and treatment of patients with hepatocellular carcinoma. Gastroenterology. 2016; 150: 835-853.

4. Hung $\mathrm{HH}$, Chiou YY, Hsia CY, et al. Survival rates are comparable after radiofrequency ablation or surgery in patients with small hepatocellular carcinomas. Clin Gastroenterol Hepatol. 2011 : 9. 79-86.

5. Lam VW, Ng KK, Chok KS, et al. Risk factors and prognostic factors of local recurrence after radiofrequency ablation of hepatocellular carcinoma. J Am Coll Surg. 2008; 207: 20-29.

6. Zhao M, Wang JP, Pan CC, et al. CT-guided radiofrequency ablation after with transarterial chemoembolization in treating unresectable hepatocellular carcinoma with long overall survival improvement. Eur J Radiol. 2012; 81: 2717-2725.

7. Morimoto M, Numata K, Kondou M, et al. Midterm outcomes in patients with intermediate-sized hepatocellular carcinoma: a randomized controlled trial for determining the efficacy of radiofrequency ablation combined with transcatheter arterial chemoembolization. Cancer. 2010; 116: 5452-5460.

8. Takuma Y, Takabatake H, Morimoto Y, et al. Comparison of combined transcatheter arterial chemoembolization and radiofrequency ablation with surgical resection by using propensity score matching in patients with hepatocellular carcinoma within Milan criteria. Radiology. 2013; 269: 927-937.

9. Xie H, Wang $\mathrm{H}, \mathrm{An} \mathrm{W}$, et al. The efficacy of radiofrequency ablation combined with transcatheter arterial chemoembolization for primary hepatocellular carcinoma in a cohort of 487 patients. PLoS One. 2014; 9: e89081.

10. Yin $\mathrm{X}$, Zhang L, Wang $\mathrm{YH}$, et al. Transcatheter arterial chemoembolization combined with radiofrequency ablation delays tumor progression and prolongs overall survival in patients with intermediate (BCLC B) hepatocellular carcinoma. BMC Cancer. 2014; 14: 849

11. Bolondi L, Burroughs A, Dufour JF, et al. Heterogeneity of patients with intermediate (BCLC B) Hepatocellular Carcinoma: proposal for a subclassification to facilitate treatment decisions. Semin Liver Dis. 2012; 32: 348-359.

12. Wada $\mathrm{H}$, Eguchi $\mathrm{H}$, Noda $\mathrm{T}$, et al Selection criteria for hepatic resection in intermediate-stage (BCLC stage B) multiple hepatocellular carcinoma. Surgery. 2016; 160(5): 1227-1235.

13. Koh YH, Choi JI, Kim HB, et al. Computed tomographic-guided radiofrequency ablation of recurrent or residual hepatocellular carcinomas around retained iodized oil after transarterial chemoembolization. Korean J Radiol. 2013; 14: 733-742.

14. Pan $\mathrm{T}, \mathrm{Li} \mathrm{XS}$, Xie $\mathrm{QK}$, et al. Safety and efficacy of transarterial chemoembolization plus sorafenib for hepatocellular carcinoma with portal venous tumour thrombus. Clin Radiol. 2014; 69: e553-e561.

15. Pan T, Xie QK, Lv N, et al. Percutaneous CT-guided Radiofrequency Ablation for Lymph Node Oligometastases from Hepatocellular Carcinoma: A Propensity Score-matching Analysis. Radiology. 2017; 282(1): 259-270.

16. Makuuchi M, Hasegawa $\mathrm{H}$, Yamazaki S. Ultrasonically guided subsegmentectomy. Surg Gynecol Obstet. 1985; 161: 346-350.

17. Sacks D, McClenny TE, Cardella JF, et al. Society of Interventional Radiology clinical practice guidelines. J Vasc Interv Radiol. 2003; 14: S199-S202.

18. Vivarelli M, Guglielmi A, Ruzzenente A, et al Surgical resection versus percutaneous radiofrequency ablation in the treatment of hepatocellular carcinoma on cirrhotic liver. Ann Surg. 2004; 240: 102-107.

19. Abu-Hilal M, Primrose JN, Casaril A, et al. Surgical resection versus radiofrequency ablation in the treatment of small unifocal hepatocellular carcinoma. J Gastrointest Surg. 2008; 12: 1521-1526.

20. Kim JW, Kim JH, Won HJ, et al. Hepatocellular carcinomas $2-3 \mathrm{~cm}$ in diameter: transarterial chemoembolization plus radiofrequency ablation vs. radiofrequency ablation alone. Eur J Radiol. 2012; 81: e189-193.

21. Yamakado K, Nakatsuka A, Takaki H, et al. Early-stage hepatocellular carcinoma: radiofrequency ablation combined with chemoembolization versus hepatectomy. Radiology. 2008; 247: 260-266. 
22. Peng ZW, Zhang YJ, Chen MS, et al. Radiofrequency ablation with or without transcatheter arterial chemoembolization in the treatment of hepatocellular carcinoma: a prospective randomized trial. J Clin Oncol. 2013; 31: 426-432.

23. Seror O, N'Kontchou G, Nault JC, et al. Hepatocellular carcinoma within milan criteria: no-touch multibipolar radiofrequency ablation for treatment-long-term results. Radiology. 2016; 280: 611-621.

24. Yin $\mathrm{L}, \mathrm{Li} \mathrm{H}, \mathrm{Li} \mathrm{AJ}$, et al. Partial hepatectomy vs. transcatheter arterial chemoembolization for resectable multiple hepatocellular carcinoma beyond Milan Criteria: a RCT. J Hepatol. 2014; 61: 82-88.

25. Vitale A, Burra P, Frigo AC, et al. Survival benefit of liver resection for patients with hepatocellular carcinoma across different Barcelona Clinic Liver Cancer stages: a multicentre study. J Hepatol. 2015; 62: 617-624.

26. Yang HJ, Lee JH, Lee DH, et al. Small single-nodule hepatocellular carcinoma: comparison of transarterial chemoembolization, radiofrequency ablation, and hepatic resection by using inverse probability weighting. Radiology. 2014; 271: 909-918.

27. Peng ZW, Lin XJ, Zhang YJ, et al. Radiofrequency ablation versus hepatic resection for the treatment of hepatocellular carcinomas $2 \mathrm{~cm}$ or smaller: a retrospective comparative study. Radiology. 2012; 262: 1022-1033.

28. Shibata T, Isoda H, Hirokawa $\mathrm{Y}$, et al. Small hepatocellular carcinoma: is radiofrequency ablation combined with transcatheter arterial chemoembolization more effective than radiofrequency ablation alone for treatment? Radiology. 2009; 252: 905-913. 